\title{
Proposal of Computer Assisted Treatment of Morphological Disorders of Mandibular Structure
}

\author{
Jarosław Sidun ${ }^{1 *}$, Urszula Mikołajczuk' ${ }^{1}$ Edyta Bitowska ${ }^{1}$ \\ 'Bialystok University of Technology, Faculty of Mechanical Engineering, ul. Wiejska 45c, 15-351 Białystok
}

\begin{abstract}
This article presents a proposal of computer assisted treatment of morphological disorders of mandibular bone structure. Cephalometric analysis according to Schwarz was conducted with the application of CorelDRAW software, generally available software for editing of vector graphics and an alternative to expensive, specialized software dedicated for computer assisted pre-operational planning. An approach to cranio- and gnatometric measurements based on cephalometric planes, lines and points is presented. Thanks to the proposed approach to computer assisted planning of treatment, cephalometric analysis is more precise and faster in comparison to traditional analysis conducted manually on a radiograph.
\end{abstract}

Keywords: computer assisted treatment, mandibula, morphological disorders

\section{Introduction}

Treatment of morphological disorders of mandibular structure is intended to improve appearance and function, achieve proper occlusion, correct proportions, facial length and mandibular rami, as well as good shape of the chin [1, 2]. The facial region is a particularly difficult one to treat, since many delicate anatomical structures are found here. Less extensive disorders can be treated orthopedically, however the majority of disorders require surgical treatment.

Before commencing treatment of disorders in the facial skeleton, the procedure must be planned thoroughly. The plan of a corrective procedure should include cephalometric analysis concerning the distribution of soft tissue and bone structures while preserving the principles of harmony and symmetry $[8,9,11]$.

The development of modern technologies, including computer assisted surgical procedures, is a very good alternative to classical planning. Thanks to computer assisted preoperative planning, it is possible to apply different imaging techniques, from classical 2D radiographs to 3D images $[5,12,13]$. This allows for more precise and faster preoperative planning of a specific patient's case in different regions of the skull. The market currently offers a wide range of software used in maxillofacial surgery, enabling cephalometric analysis and 3D modeling [3, 6, 9]. However, the cost of software is often the main factor limiting its application.

Computer assistance for surgical procedures $[4,7,8]$ :

- covers methods utilizing computer technology, applied during the preoperative period, during and after surgery, which support and facilitate a surgeon's work;

- allow for optimization of work through: 
$\checkmark$ precise planning of conducted activities, based on the patient's anatomical data, collected prior to the procedure,

$\checkmark$ precise execution of prepared plans,

$\checkmark$ improvement of the patient's safety,

$\checkmark$ minimizing interference in the patient's body.

This article presents the possibility of applying generally available and relatively inexpensive CorelDRAW vector graphics computer software for preoperative planning of the mandibular correction procedure.

\section{Research materials and methods}

Examples of radiographs, in digital form, taken before the procedure were used in this work. These images include reference points that facilitate determination of the line used to conduct all measurements required for analysis. In the case where radiographs from before and after surgical intervention are available, it is possible to compare results obtained before and after the procedure in order to determine the degree of improvement. However, it is then necessary to maintain the repeatability of obtained radiographs (they should be made using an identical technique with the same exposure parameters). Only then will it be possible to reliably assess changes resulting from undertaken treatment $[3,6,10]$.

This article presents the possibility of applying generally available CorelDRAW software for editing of vector graphical formats as a tool assisting planning of the mandibular correction procedure. Thanks to the proposed approach to computer assisted planning of treatment, cephalometric analysis is more precise and faster in comparison to traditional manual analysis. Specialized software for such analyses is more expensive by a factor exceeding ten.

\section{Research results and discussion}

The method of analyzing a lateral head radiograph according to Schwarz is one of the most widespread techniques in the world. It covers both craniometric and gnatometric measurements. This method is additionally characterized by distance measurements and the fact that the left facial profile on the radiograph is analyzed. Mose reference points are used in other analyses $[8,9$ 12].

The diagram below presents the prepared

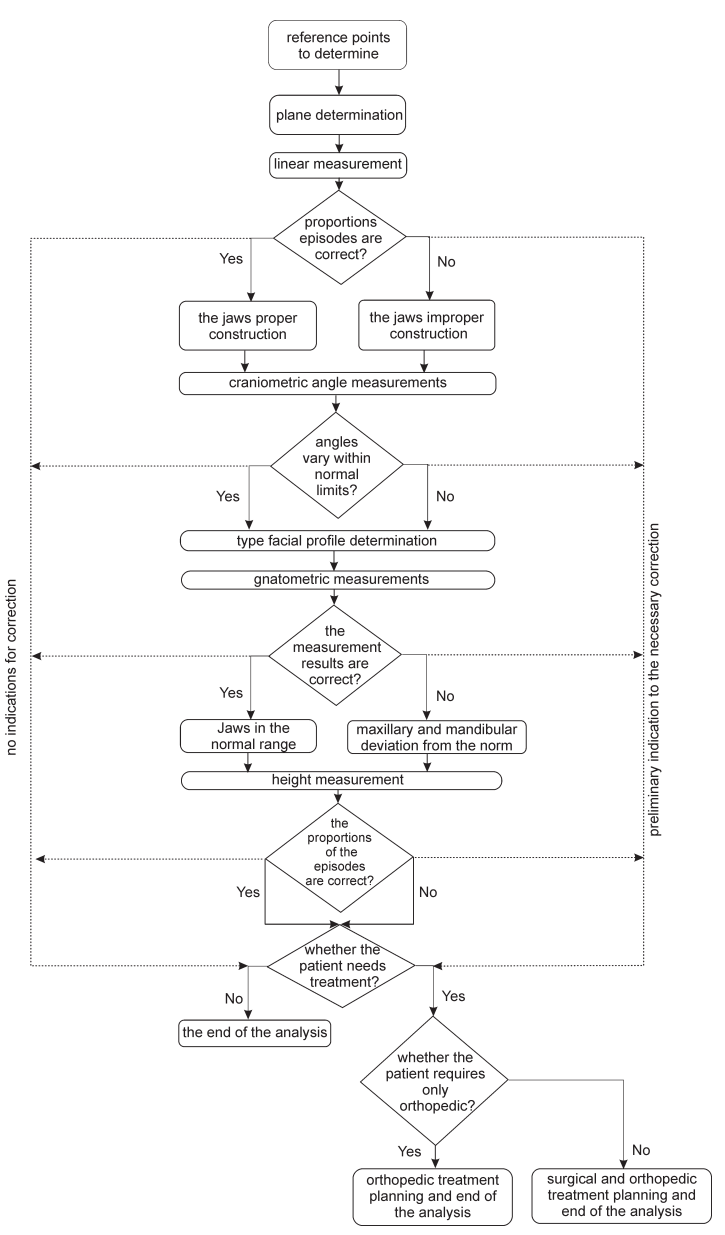

Fig. 1: Procedure algorithm during Schwarz cephalometric analysis, performed in this work.

algorithm of procedure for conducting Schwarz analysis.

Using the Object manager is a good practice when working with CorelDRAW software. This tool makes it possible to perform and inspect work in so-called layers. These are hierarchically arranged planes where the content of the higher layer covers the content of the lower layer. Work on several planes makes it possible to display layers selectively, select layers for printing and provides greater transparency of work. The current status of objects and their distribution on specific layers is also visible.

In order to facilitate determination of craniometric planes, an additional layer: Craniometric points, marked in red, was created, and it will contain only the points required for constructing planes. 
Points were constructed using the Ellipse tool. The following points were determined, in order: nasion $N$, sella turcica $S e$, orbitale Or, tragion $T$, subspinale (Downs's A point). Due to the difficulty of determining the orbital point 0 r, it was determined according to a marker introduced before the test, visible in the radiograph as a brighter point in the vicinity of the lower edge of the orbit. The tragion point $T$ was determined approximately at the peak of the condyle.

In addition, it is also possible to add a caption (comment) to each of the determined points using a text tool, as presented in the illustration below. This activity was omitted for better readability of the image subject to analysis.

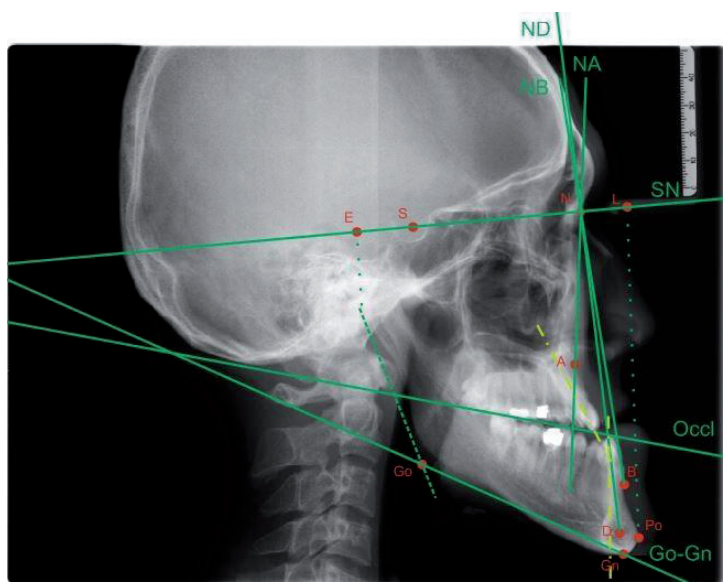

Fig. 2: Radiograph with drawn reference lines.

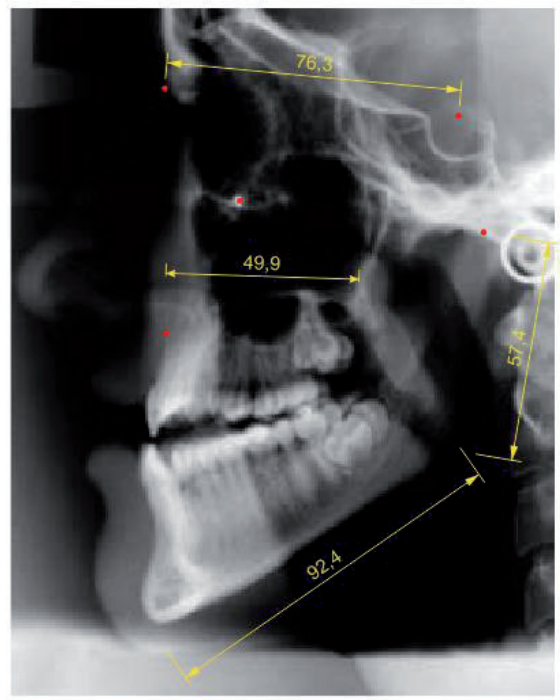

Fig. 3: Linear (left) and angular (right) dimensions.
The dotted lines determine projections of the points: posterior condyle edge (E) and pogonion $(\mathrm{L})$ on the SN line.

Determination of the angle values arising from intersection of individual planes is only based on use of the Angle dimension tool. The following angles were marked in red: facial $(F)-84.5^{\circ}$, inclination $(J)$ $94.5^{\circ}$, arrangement of temporomandibular joint $(H)$ $88.9^{\circ}$, along with the given angle dimensions.

To perform cephalometric analysis, it is necessary to determine reference points, which serve as reference points for length measurements and are then helpful in determining planes and angles in cranio- and gnatometric measurements. The following craniometric planes were introduced: anterior cranial base (NSe), Frankfort horizontal $(H)$, spinapalate $(S p P)$, nasion to Downs's A-point $(N A)$, nasion perpendicular of Dreyfus $(P n)$. Next, gnatometric planes were introduced: occlusal $(O c P)$, mandibular (MP), as well as lines: mandibular tangent to the contour of the lower mandible (MT1), tangent to the posterior mandibular ramus (MT2) (Fig. 1)

Lengths of individual segments, rounded to the nearest whole number, amounted to:

- anterior cranial base NSe length - $76 \mathrm{~mm}$;

- ength of mandibular corpus UK - $92 \mathrm{~mm}$;

- length of mandibular ramus MT2 $-57 \mathrm{~mm}$.

Comparing the ratios of measured segment lengths:

- NSe/UK = 19:23 (with correct ratio being 20:14);

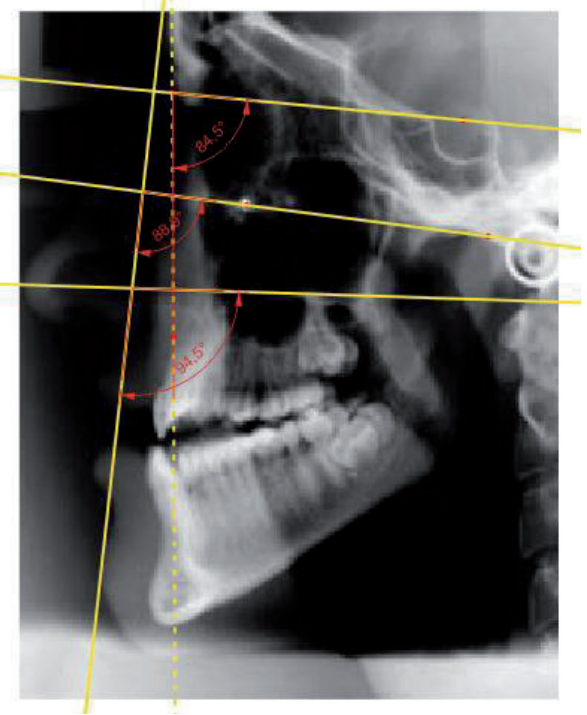




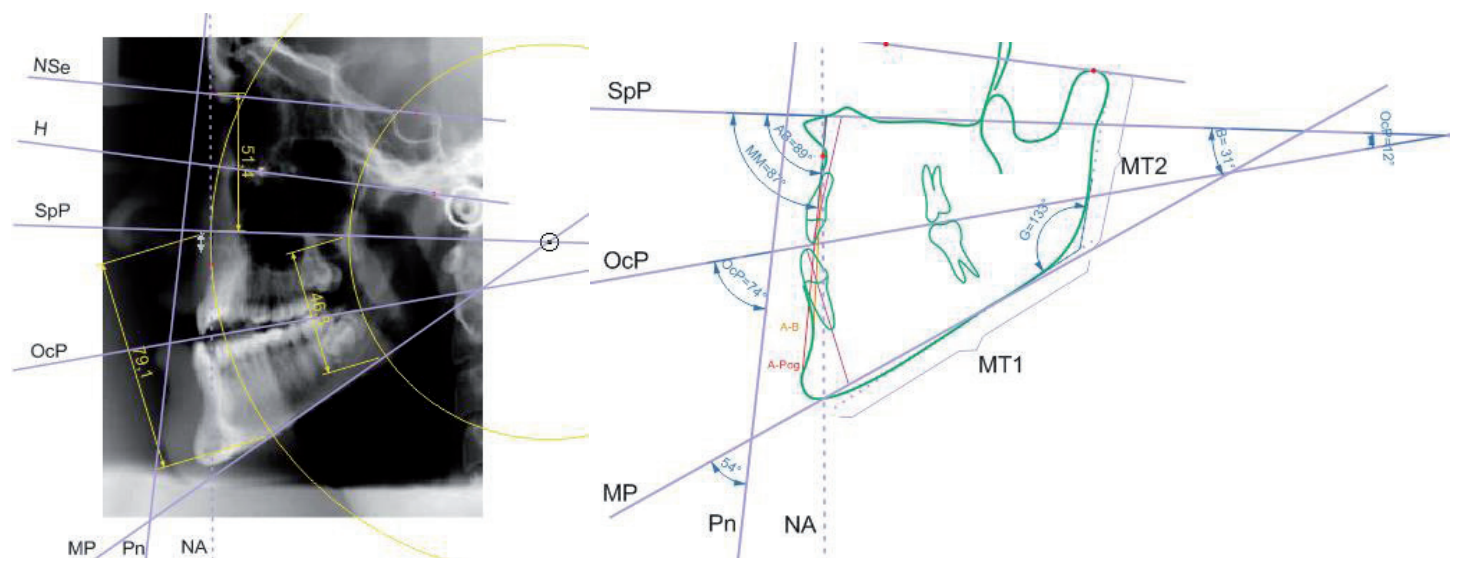

Fig. 4: Example results of cranio- and gnatometric measurements along with planes and lines indispensable for measurement.

- UKMMT2 $=92: 57$ (with correct ratio being 7:5);

excessive length of the mandibularcorpuscompared to the anterior cranial base and mandibular ramus can be concluded.

Gnatometric measurements require the introduction of additional lines, planes and angles. The layer of craniometric planes was supplemented with the planes: occlusal $(O C P)$, mandibular $(M P)$. As well as with the lines: mandibular tangent to the contour of the lower mandible $\left(M T_{1}\right)$, tangent to the posterior mandibular ramus $\left(M T_{2}\right)$, maxillary alveolar process to alveolar part of the mandible $(A-B)$, point A on maxilla to pogonion (A-Pog) and long dental axes line.

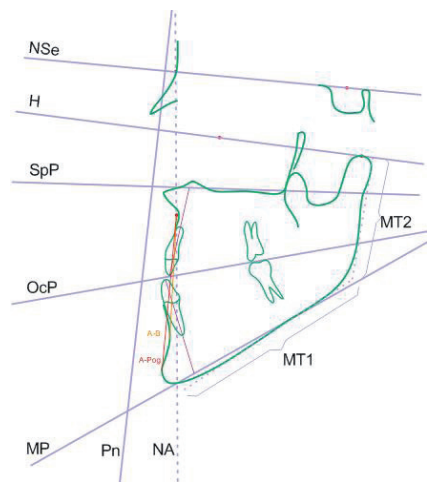

Fig. 5: Planes and lines used in craniometric and gnatometric measurements.

Next, the following gnatometric angles were determined:

- Occlusal angle OcP: craniometric with angle value of $74^{\circ}$ (below average) and gnatometric with value of $12^{\circ}$ (above average, indicating a steep occlusal plane).

- Craniometric mandibular plane, with a value of 54, significantly below average.

- Mandibulomaxillary angle $B$, with a high value of $31^{\circ}$

- Mandibular angle $G$, with a value of $133^{\circ}$, within normal limits.

- Angle $A B$, with a value of $89^{\circ}$.

- Angle $M M$, reduced, with a value of $87^{\circ}$, indicating protrusion of the mandible relative to the maxilla.

In order to present the possibilities of determining the degree of improvement achieved after corrective mandibular surgery, a compilation of results of analyses, conducted according to Schwarz's method before and after surgery, is presented below.

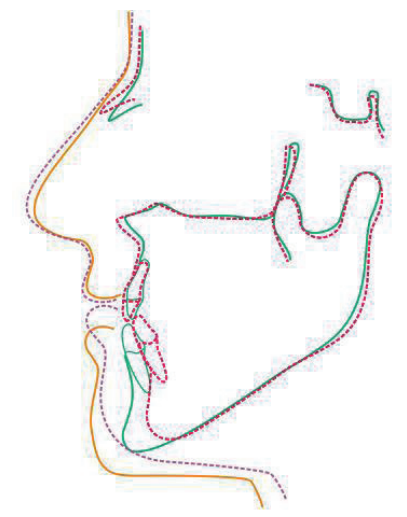

Fig. 6: Comparative compilation of the profile of bone structures and soft tissue before surgery (continuous line, green and orange) and after surgery (broken line, pink and violet).

The changes that occurred as a result of the surgical procedure can be seen in the figure above. Based on measured angle values, reduction of the angle of inclination (by $2.5^{\circ}$ ) and restoration of the correct angle value of temporomandibular joint 
arrangement $\left(90^{\circ}\right)$ can be considered.

Table 1 presents a comparison of parameter values before and after correction in reference to standard values determined by Steiner.

Tab. 1: Comparison of parameter values before and after correction with standard values.

\begin{tabular}{|c|c|c|c|c|}
\hline \multicolumn{2}{|c|}{ Parameter } & $\begin{array}{l}\text { Standard values } \\
\text { by Steiner }\end{array}$ & $\begin{array}{l}\text { Before } \\
\text { correction }\end{array}$ & $\begin{array}{l}\text { After } \\
\text { correction }\end{array}$ \\
\hline \multirow{10}{*}{ 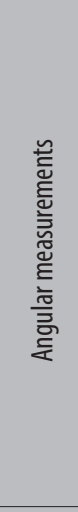 } & $\measuredangle \mathrm{SNA}$ & $82^{\circ}$ & $83^{\circ}$ & $82^{\circ}$ \\
\hline & $\measuredangle S N B$ & $80^{\circ}$ & $94^{\circ}$ & $84^{\circ}$ \\
\hline & $\measuredangle \mathrm{SND}$ & 770 & $92^{\circ}$ & $81^{\circ}$ \\
\hline & $\measuredangle A N B$ & $2^{\circ}$ & $11^{\circ}$ & $2^{\circ}$ \\
\hline & GoGn:Sn & $32^{\circ}$ & $29^{\circ}$ & $34^{\circ}$ \\
\hline & Occl:SN & $14^{\circ}$ & $16^{\circ}$ & $14^{\circ}$ \\
\hline & 1:NA & $22^{\circ}$ & $31^{\circ}$ & $29^{\circ}$ \\
\hline & 1:NB & $25^{\circ}$ & $8^{\circ}$ & $15^{\circ}$ \\
\hline & 1:GoGn & $93^{\circ}$ & $64^{\circ}$ & $80^{\circ}$ \\
\hline & 1:1 & $131^{\circ}$ & $152^{\circ}$ & $136^{\circ}$ \\
\hline \multirow{4}{*}{ 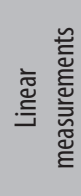 } & S:E & $22 \mathrm{~mm}$ & $23 \mathrm{~mm}$ & $23 \mathrm{~mm}$ \\
\hline & S:L & $51 \mathrm{~mm}$ & 86 mm & $54 \mathrm{~mm}$ \\
\hline & 1:NA & $4 \mathrm{~mm}$ & $9 \mathrm{~mm}$ & $8 \mathrm{~mm}$ \\
\hline & $1: N B$ & $4 \mathrm{~mm}$ & $2 \mathrm{~mm}$ & $1,5 \mathrm{~mm}$ \\
\hline
\end{tabular}

When comparing the results of measurements with standard values, one can observe that parameter values differ significantly from normal values. After correction, however, results are close to normal values. The high divergence between gnatometric dimensions 1:NA and i:NB can be corrected by means of additional orthodontic treatment. An orthodontic appliance can be used to correct the inclination of incisors, which would simultaneously improve the angular and linear parameters of these teeth.

\section{Summary and conclusions}

This article presents the possibilities of applying software for computer assistance of viscerocranial correction procedures with the utilization of standard, generally available software. The structure and topography of the facial skeleton is discussed in the theoretical part. Correct proportions preserving its harmony are also presented. Due to the structure and close vicinity of delicate structures, the facial skeleton is exposed to many deformations caused by different factors, such as post-traumatic, neoplastic and developmental changes. Techniques for imaging of deformations occurring in the area of the facial skeleton are also described. Methods of assessing viscerocranial structure are presented. Due to the high number of available methods of cephalometric analysis, the two most important methods have been described. The first of them - Schwarz analysis, is one of the most popular methods used today. The second, according to Steiner, is applied in this work. It is one of the oldest holistic analyses. This method was applied in the practical part, since, after measurements were taken, obtained results are compared to standard values of the control group (of the caucasoid type), and values of measured parameters are correct for the European population.

Based on obtained results of research, the following general conclusions were formulated:

- obtained results of cephalometric analysis make it possible to determine the degree of deformation and procedure for treatment of the facial skeleton;

- CorelDRAW software makes it possible to conduct cephalometric analysis by any method;

- the lack of automatic detection of tissue structural profiles slows down analysis, enabling more precise representation of shapes as desired by the operator, which affects the accuracy of conducted analysis;

- analysis conducted by means of computer software makes it possible to make a diagnosis more quickly and to plan correction of pathological states deviating from the norm;

- CorelDRAW software can be an effective tool assisting planning of mandibular correction procedures.

\section{References}

[1] Alley T.R.: Physiognomy and social perception. Social and applied aspects of perceiving faces. J. Maxillofac. Surg. 1988, 36, 167-172

[2] Athanasiou A.E.: Morphologic and functional implications of the surgical-orthodontic management of mandibular prognathism: a comprehensive view. Am. J. Orthod. 1993, 5, 103, 439-447

[3] Gzik M., Wolański W., Tejszerska D., Gzik-Zroska B., Koźlak M., Larysz D., Mandera M.: Application of 3D modeling and modern visualization technique to neurosurgical trigonocephaly correction in children. IFMBE Proceedings 2009, Volume 25/9, pp 68-71. 
[4] Díaz Lantada A., Del Valle-Fernández R., Lafont Morgado P., Muñoz-García J., Muñoz Sanz J. L., Munoz-Guijosa J. M., Echávarri Otero J.: Development of Personalized Annuloplasty Rings: Combination of CT Images and CAD-CAM Tools. Annals of Biomedical Engineering. 2/2010, Volume 38, Issue 2, pp 280-290.

[5] Durgesh N. Bailoor (red.), K. N. Fundamentals of Oral Medicine and Radiology. Jaypee Brothers Medical Publishers, New Delhi 2005.

[6] Gzik M., Wolański W., Tejszerska D., Gzik-Zroska B., Koźlak M., Larysz D., Mandera M.: Application of 3D modeling and modern visualization technique to neurosurgical trigonocephaly correction in children. In: Dössel O., WSchlegel. C.: World Congress on Medical Physics and Biomedical Engineering, September 7 - 12, 2009, Munich, Germany. IFMBE Proceedings 2009, Volume 25/9, pp 68-71. Springer, Berlin Heidelberg 2009.

[7] Huang Z., Chen Z.: Three-dimensional finite element modeling of a maxillary premolar tooth based on the microCT scanning: A detailed description. Journal of Huazhong University of Science and Technology [Medical Sciences]. 10/2013, Volume 33, Issue 5, pp 775-779.

[8] Liu Y., Bai S., Zhang G., Liao Q., Lu H.: FPCAD: 3D computeraided system for facial prosthetics using VTK. In: CranioMaxillofacial Surgery. International Journal of Computer Assisted Radiology and Surgery. 6/2013, Volume 8, Issue 1
Supplement, p 91.

[9] Maal T., Verhamme L., Van Lindert E., Borstlap W., Bergé S.: Computation of an average cranial shape of three to twelve months old infants using 3D stereophotogrammetry. In: Cranio-Maxillofacial Surgery. International Journal of Computer Assisted Radiology and Surgery. 6/2012, Volume 7, Issue 1 Supplement, p 119.

[10] Michalikova M., Bednarcikova L., Petrik M., Zivcak J. et al.The Digital Pre-Operative Planning of Total Hip Arthroplasty. Acta Polytechnica Hungarica, Volume: 7, Issue: 3, pp. 137152

[11] Molcanyi M., Molcanyi T., Zivcak J., Hudak R.: Original angular instrument set for ventral ostheosynthesis of odontoid fracture in patients with barrel chest. European Spine Journal 17(11), 2008.

[12] Souccar N. M., Kau C. H., Weinberg S. M.: Digital Three-Dimensional Photogrammetry: Craniofacial Applications to Facial Growth, Orthognathic and Reconstructive Surgery, and Morphometrics. In: Preedy V. R.: Handbook of Anthropometry. Springer, New York 2012.

[13] Wilde F., Cornelius C.-P., Schramm A.: Computer-assisted mandibular reconstruction with CAD/CAM fabricated patient-specific reconstruction plates: A cadaver feasibility study. In: Cranio-Maxillofacial Surgery. International Journal of Computer Assisted Radiology and Surgery. 6/2013, Volume 8, Issue 1 Supplement, pp 87-88. 